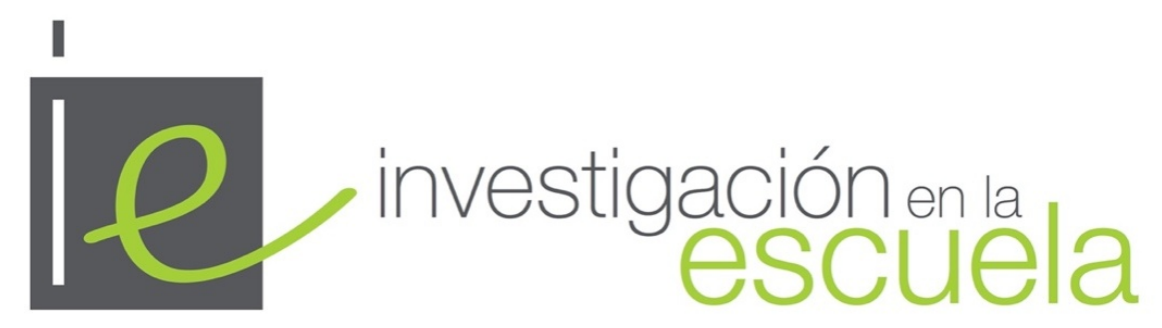

Revista de Investigación e Innovación Educativa nº 101, 2020 | e-ISSN 2443-9991

\title{
Testimonios de víctimas en el módulo educativo Adi-adian. Una mirada desde la didáctica de las ciencias sociales y la educación patrimonial
}

\author{
Victims' testimonies in the adi-adian educational module. A view from the didactics of social sciences and heritage \\ education
}

Lic. Olatz Aranguren-Juaristi es Profesora Laboral Interina en la Facultad de Educación, Filosofía y

iD Antropología de la Universidad del País Vasco UPV/EHU (España)· olatz.aranguren@ehu.eus https://orcid.org/0000-0003-2451-5829

Dra. Dorleta Apaolaza-Llorente es Profesora Laboral Interina en la Facultad de Educación y Deporte de la

iD Universidad del País Vasco UPV/EHU (España)·dorleta.apaolaza@ehu.eus https://orcid.org/0000-0003-02281478

Dra. Berta Echeberria Arquero es Profesora Laboral Interina en la Facultad de Educación, Filosofía y

iD Antropología de la Universidad del País Vasco UPV/EHU (España)·berta.echeberria@ehu.eus https://orcid.org/0000-0002-0356-7833

Dra. Naiara Vicent es Profesora Laboral Interina en la Facultad de Educación, Filosofía y Antropología de la

(iD Universidad del País Vasco UPV/EHU (España)·naiara.vicent@ehu.eus https://orcid.org/0000-0002-26780098

Resumen. Este trabajo presenta un estudio preliminar llevado a cabo durante la implementación del módulo educativo Adi-adian en los grados de Educación Infantil y Primaria. En él se acerca al alumnado la historia reciente del País Vasco a través de testimonios de víctimas. A través de una ficha de registro, se ha obtenido información de manos del profesorado participante. Ésta ha corroborado el alto impacto ejercido por los testimonios, a través de los cuales el alumnado ha conocido y se ha interesado por el tema, impulsando una reflexión crítica. También se han reconocido las posibilidades que ofrece para trabajar temas controvertidos en la formación inicial del profesorado y, aunque no se ha abordado de manera explícita, la educación emocional.

Abstract. This work presents a preliminary study carried out during the implementation of Adi-adian educational module in Early Childhood and Primary Education university degrees. In this module, the recent history of the Basque Country is brought closer to students through the testimony of victims. Through a registration form, data has been obtained from the participating teachers. This information has confirmed the high impact exerted by the testimonies, through which the students have known and increase their interest on the subject, encouraging a critical reflection. Besides, the possibilities it offers to work with controversial issues in initial teachers' education and, although not explicitly addressed, emotional education has also been recognized.

\section{Palabras clave $\cdot$ Keywords}

Patrimonio inmaterial, memoria colectiva, didáctica de las ciencias sociales, educación para la ciudadanía, conflicto armado, temas controvertidos, emociones, programa educativo.

Intangible heritage, collective memory, social studies, citizenship education, armed conflict, controversial issues, emotions, education program. 


\section{Introducción}

El tratamiento de las cuestiones relevantes en el aula de ciencias sociales ha de ser una de las líneas de trabajo en la formación inicial del profesorado. Una de estas cuestiones que afecta directamente a nuestro alumnado es la que se ha generado tras la tregua y posterior disolución de ETA.

La juventud no ha vivido los momentos de mayor crispación y muchos no conocen lo que ha sucedido (Usón, 2017). Ante esta situación y la diversidad de relatos que existen sobre lo ocurrido, resulta importante ofrecer a los más jóvenes el derecho a conocer. A través del "Módulo Educativo Adi-adian. Aprendizajes de dignidad humana, convivencia y empatía mediante una experiencia de escucha de testimonios de víctimas", promovido por el Gobierno Vasco (2013), se pretende dar a conocer a la juventud una parte de esta etapa de nuestra historia reciente.

El departamento de Didáctica de las Ciencias Sociales de la Universidad del País Vasco comenzó a implementar el módulo a pequeña escala, convirtiéndose durante el curso 2018-2019 en una propuesta curricular asumida por todo el departamento. El módulo Adi-adian tiene por objetivos ofrecer una experiencia y abordar la empatía, la dignidad humana, los derechos humanos y la convivencia, teniendo como eje principal la escucha de testimonios de víctimas (Gobierno Vasco, 2013).

En este trabajo se muestra un estudio preliminar que busca conocer el impacto ejercido por los testimonios en el alumnado, los procesos de patrimonialización desarrollados y las posibilidades que estos testimonios ofrecen para abordar en el aula temas controvertidos, así como trabajar la educación emocional.

\section{Marco teórico}

Ya en 2011 la Confederación de Sociedades Científicas de España criticaba el poco peso que se había dado a la exploración de fenómenos y la indagación en las aulas, perviviendo todavía un mero método «factual y reproductivo». Si trasladamos esta crítica al ámbito de las Ciencias Sociales, basado en las competencias ciudadanas, se debería cambiar el método para poder promover el pensamiento crítico con el desarrollo de habilidades cívicas. Esto pasaría por demostrar al alumnado la unión de las ciencias con la realidad en la que viven: estudiar su propia realidad, para poder elaborar su visión crítica del mundo y, por ende, su competencia ciudadana y científica. La enseñanza basada en los conflictos sociales candentes o temas controvertidos, será una de las aproximaciones más destacadas para lograr este objetivo (López Facal y Santidrián, 2011).

Este tipo de enseñanza sería aquella que se refiere a temas estructurados alrededor de preguntas que tienen más de una respuesta, que obligan a trabajar desde una multiplicidad de perspectivas. Estas requieren por parte del alumnado el construir su propio discurso haciendo uso del espíritu crítico y de su capacidad para contrastar distintos puntos de vista (Goldberg y Savenije, 2018). Son varias las líneas teóricas que reivindican este tipo de enseñanza dentro del currículum de Ciencias Sociales. No solo por la posibilidad de acercar el aula a la realidad, sino porque se trabaja con algunas de las bases de la educación democrática, como son el debate y el contraste de opiniones, la argumentación y el consenso. En última instancia, es pensar "la enseñanza de las ciencias sociales como herramienta para el cambio social" (Santisteban Fernández, 2019). Podríamos concluir, por tanto, que sería una de las estrategias más idóneas para trabajar la ciudadanía y sus valores, como el respeto por los derechos humanos (Pineda-Alfonso, 2015).

En todo caso, esta aproximación requiere de un esfuerzo por parte del profesorado. Son temas sobre los que no existe un consenso y pueden generar conflictos, lo que exige una neutralidad por parte del profesor. Un concepto complejo que puede tener distintas aproximaciones, ya que dependiendo del grado de neutralidad del profesorado el acercamiento al hecho será distinto, al igual que los resultados de aprendizaje (López Facal y Santidrián, 2011). En muchas ocasiones, cuesta lograr esta neutralidad, más aún, cuando en la formación inicial del profesorado generalmente no está extendida esta forma de trabajo. El temor a manifestar su opinión lleva al profesorado a rechazar la inclusión de este tipo de cuestiones en el aula, siendo los temas identificados como más complicados aquellos relacionados con la violación de los derechos fundamentales de las personas (Toledo Jofré, Magendzo, Gutiérrez Gianella, y Iglesias Segura, 2015).

El acercamiento a la memoria histórica traumática es uno de estos temas controvertidos. Si bien es difícil dar una definición universal del concepto de memoria colectiva por las diferentes ópticas que aportan las distintas ciencias (Wertsch y Roediger, 2008), desde la óptica de la Didáctica de las Ciencias Sociales, consideramos que el pensamiento histórico puede jugar un papel importante en la reconstrucción de la memoria colectiva. Las emociones colectivas y personales están presentes en el desarrollo de los procesos de enseñanza-aprendizaje de la historia, constituyendo parte de la formación y enriqueciendo la experiencia 
educativa (Pagés-Blanch, Martínez-Valcárcel y Cachari-Aldunate, 2014). En otro ejemplo, desde la dimensión de la construcción identitaria, los relatos que se crean con este fin a partir de nuestros recuerdos individuales y colectivos son complejos. En su creación entran en juego conexiones entre discursos históricos, vivencias colectivas e individuales, recuerdos autobiográficos e identidades complejas. Podemos afirmar que la identidad tiene una concepción narrativa histórica según la cual las personas y colectivos construyen sus relatos sobre sí mismos mediante sus actuaciones, y que la participación en un escenario cultural concreto puede transformar los modos de construcción de las identidades colectivas e individuales (Marco Macarro y Sánchez Medina, 2008).

Por otro lado, la memoria histórica supone un reto en las sociedades actuales, en un equilibrio entre la globalidad y la construcción de las identidades e historias nacionales, cuestionando o reinterpretando las versiones que habían sido dadas. En este punto, la enseñanza de la historia en los centros educativos jugará un papel muy importante (Carretero, 2007). Podemos decir, sin equivocarnos, que en los últimos años se ha vivido una revitalización del concepto de la memoria en las aulas de enseñanza de la historia. Historia y memoria comparten el mismo objetivo vinculado a la elaboración del relato del pasado, y sus puntos de unión son aún más claros cuando se incide en la importancia de las vivencias de los testimonios para el estudio de la historia reciente. Los mismos son fuentes orales que permiten sacar a la luz aspectos de la realidad que antes quedaban deslavados o desaparecidos por la historia estructural y de larga duración (González y Pagés, 2014).

Si entendemos el patrimonio como una construcción social constituido por los bienes representativos de cada sociedad y que tiene como último objetivo el mantenimiento y la transmisión de la memoria colectiva, podemos decir que el patrimonio es por sí mismo un registro de la memoria social (Marcos Arévalo, 2010). Pero también podemos considerar que la memoria es, per se, un elemento patrimonializado. No solo por ser una construcción social sino también por su carácter de fenómeno representativo que dota de identidad a una comunidad y explica su propia evolución y esencia. Recordemos la diversidad del objeto patrimonial, que escapa del formato solamente físico. Es la propia sociedad la que define lo patrimonial, siendo los valores derivados de las experiencias y las relaciones que la sociedad entabla con el objeto patrimonial lo que lo convierten en patrimonio (Fontal y Martínez, 2017).

Fontal (2003) considera que el proceso de patrimonialización pasa por comprender-respetar-valorarcuidar-disfrutar-trasmitir. Un proceso complejo y poco neutral porque cada individuo juega un papel con sus propias emociones y creencias. La complejidad aumenta en aquellos procesos comunitarios. Esto es, hay que entender que el compartir una identificación de un suceso como patrimonio colectivo no significa obligatoriamente que todo el grupo comparta las mismas emociones hacia el mismo. Y si a esto le añadimos el proceso vivo que es la definición de patrimonio, siempre sujeto a un intercambio de opiniones y debate, se ha de comprender que los sujetos pueden cambiar sus posiciones e incluso acercarse a las de otros individuos (Rana, Willemsen, y Dibbits, 2017). Esto convierte en complejo la construcción de la memoria como patrimonio común, porque aúna distintas perspectivas, emociones y experiencias.

Por tanto, las emociones y la memoria colectiva juegan un papel fundamental en la definición de patrimonio, en tanto que es el resultado de un sentimiento de conexión compartida con ese elemento patrimonializado. Cuanto mayor información tengamos sobre el elemento patrimonial, más clara será la implicación emocional con el mismo (Lucas y Estepa, 2017), lo cual traerá su consiguiente reconocimiento como símbolo identitario digno de ser trasmitido a las próximas generaciones. Así, la introducción de cuestiones patrimoniales o referidas a la memoria colectiva, ofrecen la posibilidad de trabajar la educación emocional en el aula. Concretamente trabajar la Educación patrimonial desde un enfoque simbólicoidentitario permite traer al aula el trabajo de las emociones a la vez que se ahonda en la construcción de identidad del individuo y la valoración-conservación del objeto patrimonial (Gómez Hurtado y Cuenca López, 2017). Sin embargo, hay estudios que indican que, si bien el profesorado valora positivamente la importancia de ésta, la mayoría no tienen los conocimientos necesarios para trabajarla desde las evidencias científicas (Bidaurratzaga y Bustillo, 2020).

Volviendo a la memoria traumática, Arnoso, Páez, Bobowik y Basabe (2018), describen en su trabajo el consenso a la hora de identificar a la violencia como acontecimiento central en las memorias colectivas. Igualmente, señalan el impacto emocional generado por acontecimientos violentos, destacando el papel de las emociones en la creación y el mantenimiento de la memoria colectiva, si bien, como señalan los/as autores/as, numerosos estudios muestran la existencia de visiones sesgadas de la realidad, dirigidas a favorecer la idea de que el grupo externo es el agresor y el propio es la víctima. Por eso, reivindican que una de las competencias que debe abordarse desde la enseñanza de la historia debe ser la capacidad de comprensión de los diversos factores que intervienen, pero también la necesidad de llevar al aula testimonios silenciados, especialmente en los casos en que se ha dado un conflicto. 
En el caso del módulo Adi-adian (Gobierno Vasco, 2013), se entiende el testimonio de las víctimas como pilar necesario para todo proceso de reconciliación. Se plantea cómo las víctimas de violencia posibilitan o encauzan la construcción de la memoria colectiva. Los testimonios de las víctimas y su punto de vista son básicos para la construcción de un diálogo empático y su mirada puede estimular procesos de reconciliación y desarrollar una concepción de la política que no se base en la violencia (Bilbao y Etxeberria, 2005). En concreto, el módulo Adi-adian tal y como se ha implementado en el departamento de Didáctica de las Ciencias Sociales, incorpora una sesión de sensibilización y dos sesiones de escucha a víctimas de diversa índole, a las que el profesorado del departamento a incorporado una sesión de reflexión. Además, algunos/as docentes han complementado la propuesta con la realización de un proyecto de investigación.

En el presente estudio, a través de las implementaciones de Adi-adian desarrolladas por el departamento de Didáctica de las Ciencias Sociales, se pretende:

1. Conocer, a través del profesorado implicado, el impacto ejercido por los testimonios de las víctimas en el alumnado.

2. Conocer, a través del profesorado, si los testimonios de las víctimas son considerados patrimonio por el alumnado.

3. Conocer las percepciones del profesorado sobre las posibilidades que ofrece Adi-adian para trabajar las ciencias sociales a través de temas controvertidos.

4. Conocer las percepciones del profesorado sobre la posibilidad que ofrece Adi-adian para poder trabajar la educación emocional.

\section{Método}

Este es un estudio cualitativo de tipo descriptivo, desarrollado bajo el marco de un proyecto de innovación pedagógica cuyo objetivo es conocer y mejorar las implementaciones desarrolladas por el departamento de Didáctica de las Ciencias Sociales con relación al módulo Adi-adian. Para ello se ha utilizado la percepción de los docentes que lo han integrado en su asignatura durante el curso académico 2018-19.

Como herramienta para la recogida de datos se ha creado por los miembros del equipo de investigación una ficha de registro a cumplimentar por los/as docentes que integraron Adi-adian en sus sesiones universitarias. Por lo tanto, la información se obtiene a través de la observación participante del profesorado y su posterior narración de los acontecimientos. Además, en los casos en los que ha sido necesario, la información recogida en las fichas de registro se ha complementado con una entrevista personal.

La herramienta está formulada a través de preguntas abiertas, buscando identificar: las dinámicas y tareas llevadas a cabo en el aula; los recursos utilizados; el ambiente vivido durante las sesiones y la valoración de las mismas; la percepción en relación a las sesiones de escucha de testimonios; el cumplimiento de los objetivos educativos; las posibilidades que ofrece el módulo a la formación de futuros docentes; la motivación tanto del profesorado como del alumnado en torno al tema tratado; la influencia ejercida por la propuesta entre los/las docentes y el alumnado; y por último, la identificación de posibles mejoras en la implementación del módulo.

A principios de abril de 2019, tras haber finalizado en todas las asignaturas la implementación del módulo Adi-adian, se envió por correo electrónico la ficha de registro al profesorado implicado. Durante las siguientes semanas, cada docente se encargó de rellenarla de manera individual. Posteriormente, las dudas sobre la cumplimentación o la posterior interpretación de los datos recogidos fueron resueltas a través de entrevistas realizadas por teléfono.

Tras una lectura pormenorizada de las fichas de registro, se ha analizado el contenido y las narraciones expuestas en las mismas (López Noguero, 2002). Así, se han codificado los participantes (Docente 1, Docente 2...) y sus respuestas extrayendo aquellas frases representativas que ofrezcan pistas sobre las cuestiones a analizar. De cara a cumplir con los objetivos, en relación al alumnado, se ha prestado atención a: cuestiones relacionadas con la satisfacción; asimilación de contenidos históricos; actitudes empáticas, críticas y referidas a la vulneración de los derechos humanos; trasmisión de conocimientos; o emociones vividas. En cuanto al profesorado, se ha reparado en su percepción en torno al tratamiento de temas controvertidos en el aula, gestión de conflictos o educación emocional (Tabla 1). 
Tabla 1

Objetos de análisis en relación a los objetivos de la investigación

\begin{tabular}{|c|c|}
\hline Objetivos de la investigación & Objeto de análisis \\
\hline \multirow{6}{*}{$\begin{array}{l}\text { Conocer, a través del profesorado implicado, el } \\
\text { impacto ejercido por los testimonios de las víctimas } \\
\text { en el alumnado. }\end{array}$} & Satisfacción \\
\hline & Conocimiento del contexto histórico \\
\hline & Espíritu crítico \\
\hline & Empatía \\
\hline & Actitud ante la vulneración de Derechos \\
\hline & Humanos \\
\hline $\begin{array}{l}\text { Conocer, a través del profesorado, si los testimonios } \\
\text { de las víctimas son considerados patrimonio por el } \\
\text { alumnado. }\end{array}$ & Trasmisión \\
\hline \multirow{2}{*}{$\begin{array}{l}\text { Conocer las percepciones del profesorado sobre las } \\
\text { posibilidades que ofrece Adi-adian para trabajar las } \\
\text { ciencias sociales a través de temas controvertidos. }\end{array}$} & Temas controvertidos \\
\hline & Gestión de conflictos \\
\hline \multirow{2}{*}{$\begin{array}{l}\text { Conocer las percepciones del profesorado sobre la } \\
\text { posibilidad que ofrece Adi-adian para poder trabajar } \\
\text { la educación emocional. }\end{array}$} & Emociones vividas por el alumnado \\
\hline & Educación emocional \\
\hline
\end{tabular}

Dado que la mayor parte de la docencia ofrecida por el departamento se oferta en vasco, y en cuanto que este es el idioma de comunicación entre el profesorado del mismo, tanto la ficha de registro como la información recogida se ha ejecutado en este idioma. Por lo tanto, para la realización de este artículo se ha procedido a la traducción al castellano de algunas de las frases recogidas en los resultados.

\subsection{Muestra}

La muestra está compuesta por 14 docentes del departamento de Didáctica de las Ciencias Sociales, repartidos entre las tres facultades que ofrecen estudios de educación en la Universidad del País Vasco. Estos han implementado el módulo Adi-adian en diversas asignaturas de Ciencias Sociales recogidas en los grados de Educación Infantil y Educación Primaria, así como del Máster de Secundaria (Tabla 2).

\section{Tabla 2}

Datos generales de la implementación del programa Adi-adian durante el curso 2018-2019

\begin{tabular}{|c|c|c|c|c|}
\hline Titulación & Asignaturas & Curso & $\begin{array}{l}\mathrm{N}^{\mathrm{o}} \text { de } \\
\text { docentes } \\
\text { implicados }\end{array}$ & $\begin{array}{l}\mathrm{N}^{\circ} \text { de } \\
\text { alumnos/as }\end{array}$ \\
\hline \multirow{2}{*}{$\begin{array}{l}\text { Grado de } \\
\text { Educación Infantil }\end{array}$} & $\begin{array}{l}\text { Conocimiento del } \\
\text { Medio Social y } \\
\text { Cultural }\end{array}$ & $2^{\circ}$ curso & $\begin{array}{l}4 \\
\text { (Docentes 1, 4, } \\
5 \text { y 9) }\end{array}$ & 156 \\
\hline & $\begin{array}{l}\text { Taller de } \\
\text { Experimentación en } \\
\text { el entorno social }\end{array}$ & $4^{\circ}$ curso & $\begin{array}{l}2 \\
\text { (Docentes } 8 \mathrm{y} \\
11 \text { ) }\end{array}$ & 60 \\
\hline $\begin{array}{l}\text { Grado de } \\
\text { Educación Primaria }\end{array}$ & $\begin{array}{l}\text { Ciencias sociales y su } \\
\text { didáctica I }\end{array}$ & $1^{\circ}$ curso & $\begin{array}{l}7 \\
\text { (Docentes 3, 6, } \\
7,10,12,13 \mathrm{y} \\
14)\end{array}$ & 544 \\
\hline $\begin{array}{l}\text { Máster Universitario } \\
\text { en Formación de } \\
\text { Profesorado de } \\
\text { Educación } \\
\text { Secundaria }\end{array}$ & $\begin{array}{l}\text { Ciencias Sociales y } \\
\text { Educación }\end{array}$ & - & $\begin{array}{l}1 \\
\text { (Docente } 2)\end{array}$ & 47 \\
\hline
\end{tabular}

La distribución de la muestra por género es equitativa, con un 50\% $(n=7)$ de mujeres y un $50 \%(n=7)$ de hombres. En cuanto a la experiencia, el espectro es amplio y va desde docentes recién incorporados a la universidad hasta profesorado con más de 35 años de experiencia en su último año previo a la jubilación. 


\section{Resultados}

A través de la información ofrecida por el profesorado se han conocido algunos aspectos relativos a las cuestiones que analizamos en este trabajo.

En concreto, en relación con las sesiones de escucha de testimonios de víctimas, como señala el Docente 1, se valora muy positivamente "la oportunidad ofrecida para recibir el testimonio directo de las víctimas que han sufrido la violencia", siendo esta una opinión generalizada entre el profesorado. El grupo de docentes destaca la implicación del alumnado en las mismas, indicando que este "mostró un gran interés sobre lo escuchado y la gente se mostró emocionada" (Docente 2).

Una buena manera de conocer el impacto generado por estas sesiones es a través de la ronda de preguntas que se abre una vez la víctima ha contado su historia. En este sentido, si bien hay dos profesores que indican poca participación por parte del alumnado (Docentes 3 y 10), la normalidad es que "hubo preguntas tras los testimonios" (Docente 4). Además de algún agradecimiento, la mayoría de los comentarios fueron "en torno a las emociones de las víctimas o preguntas encauzadas a poder entender mejor lo ocurrido" (Docente 8). Se ha descrito la curiosidad que nuestro alumnado siente por conocer los sentimientos de estas víctimas y, entre las emociones recogidas, el odio es la que más veces se cita. Los/las alumnos/as preguntan "cómo vivirlo, cómo evitarlo..." (Docente 6). Según varios docentes (Docentes 1, 5,7 y10) quieren saber cómo han sido capaces de superarlo, de perdonar... También preguntan por la superación de la tristeza Docente 11).

Más allá de los testimonios, las narraciones del profesorado también reconocen el poder emocional del módulo ("es un programa imprescindible para [...] profundizar en la educación emocional; en cada sesión se mueve algo dentro" (Docente 11) o del tema a tratar ("nos sitúa ante un problema social cercano trabajando conjuntamente el aspecto emocional" (Docente 12). Un docente añade que al alumnado "le ha servido para conocer de cerca las nefastas consecuencias que ha acarreado el conflicto vasco y hacer ese conocimiento a través de las emociones", subrayando que "así se consigue un impacto mayor" (Docente 7). Sin embargo, son pocos los docentes que reconocen las posibilidades que la experiencia ofrece para "profundizar en la educación emocional (Docente 8), aunque algunas de las propuestas incorporadas por los docentes del departamento pueden ser entendidas dentro de la misma. Es el caso de una tarea en la que "Individualmente, en grupos pequeños y posteriormente entre toda la clase se le pidió que resaltasen tres emociones o ideas surgidas a partir de la experiencia vivida. Se escribieron las emociones o ideas del alumnado en la pizarra y se intentó hacer una clasificación (emociones, puntos fuertes, retos para el futuro...)" (Docente 8).

A diferencia de la escasez de comentarios en torno a la educación emocional, la propuesta es reconocida de manera generalizada como "un escenario inmejorable para trabajar la empatía" (Docente 11). Se ratifica que "a los alumnos se les abren los ojos" (Docentes 1 y 4). El alumnado "recibe una información directa sobre una realidad desconocida, conoce y realiza una valoración sobre lo ocurrido en sus respectivos pueblos o ciudades, contrasta los prejuicios, supera los discursos de dirección única o por lo menos escucha opiniones de los demás" (Docente 3). Entre los/las docentes, se afirma que "es necesario un programa de tales características tanto para trabajar la empatía histórica como la social, para que no se pierda la memoria cercana" (Docente 6) que impulsa "el conocimiento de un capítulo de la historia vasca contemporánea, que aporta una oportunidad para que el alumnado conozca un tema desconocido para muchos, pero sustancial" (Docente 1).

Son numerosas las afirmaciones que indican que la propuesta ha servido para dar a conocer un tema desconocido por la juventud, así como que esta se ha sentido motivada por el mismo. Sin embargo, varios docentes afirman que la propuesta se ha quedado en un acercamiento o introducción al tema. Una de las cuestiones más citadas entre las propuestas de mejora es la necesidad de trabajar el contexto histórico con el alumnado. Esta percepción se ha recogido también por parte de una docente que implementa el módulo dentro de un proyecto más amplio en el que se lleva a cabo una tarea de investigación. Esta añade que el proyecto desarrollado "les ha servido [a los alumnos] para identificar sucesos relacionados tanto con el conflicto vasco como con la guerra de 1936 y para poder colocar esos sucesos en el contexto, en el espacio y en el tiempo que les corresponde. Para poder realizar un acercamiento. Pero, el proyecto queda bastante suelto si no se elabora a la vez un trabajo teórico (Docente 7)".

En el caso de "los que más saben", un profesor indica que "se sienten incómodos con algunos aspectos del programa porque se sienten interpelados" (Docente 3). Lo cual le lleva a "confirmar la necesidad de la actividad". De hecho, en el caso del grupo del máster, en el que "los/las alumnos/as, por edad, tienen su opinión asentada" y conocen la problemática de primera mano, se pasó de ofrecer una actitud negativa hacia el tratamiento del tema a una aceptación. El profesor que guiaba al grupo señala que "la actitud negativa cambió en las sesiones de víctimas y el alumnado se mostró bastante emocionado" (Docente 2). En relación 
con este tipo de actitud, también una profesora narra cómo "Algunos vienen con prejuicios políticos concretos y han valorado como enriquecedor escuchar lo que ha contado una persona de distinto signo" (Docente 4).

Volviendo a los/las más jóvenes, a pesar de que según la percepción del profesorado "no se habla mucho en sus casas" sobre el tema, la propuesta didáctica les ha llevado a hacerlo (Docente 8). Si bien tres docentes (Docentes 3, 7 y 10) señalan haber exigido la realización de entrevistas, en un caso en el que no se han exigido este tipo de tareas también se ha declarado que "los alumnos han hablado con la familia sobre ETA o sobre la sociedad vasca" (Docente 8). Se subraya que "la idea del diálogo intergeneracional es muy interesante y que iniciativas similares ofrecen la oportunidad a los alumnos de escuchar testimonios de personas más adultas y poder hablar con ellos", conociendo así "las condiciones de vida de sus padres y de sus abuelos" (Docente 3); recibiendo "una semilla para el futuro" (Docente 3).

Según las percepciones del profesorado, se sostiene que "además de desarrollar un tema no tratado con anterioridad [en clase]se aprenden nuevos recursos para poder trabajar este tema tan complicado" (Docente 5). Se añade que el trabajo realizado sirve para "traer al aula la enseñanza-aprendizaje de los temas relevantes" (Docente 3). Esta es una idea generalizada entre prácticamente la totalidad del profesorado, quien lo ha vivido como una experiencia válida desde un punto de vista personal, para "empezar a reflexionar sobre cómo debemos tratar estos temas" en el aula (Docente 7). Pero también como una oportunidad para nuestro alumnado: "si el profesorado del futuro se da cuenta de la dificultad que conlleva y reflexiona sobre ello, va a poseer unos criterios previos para cuando lo tenga que trabajar en clase y lo trabajará de otra manera" (Docente 2).

Varios docentes destacan que "esta actividad genera grandes aportaciones a la formación del profesorado". Al haberse tratado un tema controvertido, en varias narraciones se recoge su vinculación con el tratamiento de los conflictos (Docentes 4, 5, 7 y 10). En concreto, una profesora reconoce las posibilidades que la propuesta ofrece para un "acercamiento al conflicto", el cual "debe ser trabajado en el aula de cara a la convivencia" (Docente 7). En este sentido, otra docente señala que "sobre todo ayuda a incorporar en la formación la idea de la transformación de los conflictos, que afrontar los conflictos puede ser una vía de enriquecimiento personal y como los alumnos son futuros profesores, es de pensar que se transmitirá a los niños y niñas el punto de vista positivo de los conflictos" (Docente 4). Aunque, hay quien critica que debería de insistirse más al alumnado para que "pierda el miedo al conflicto" y reconozca que "abordarlo puede ser enriquecedor" (Docente 4).

Como señala una docente, "Las víctimas en general han subrayado el sinsentido de utilizar la violencia y creo que es una de las ideas que han quedado más claras" (Docente 4), lo que ha traído como consecuencia la construcción por parte del alumnado de "un discurso contra la violencia" (Docentes 4 y 10). Además, se reconoce que la propuesta "impulsa una convivencia basada en los derechos humanos para el futuro" (Docente 11). "Los alumnos han podido reflexionar sobre los derechos y obligaciones que tenemos como ciudadanos, han trabajado profundamente los valores y los derechos humanos a través de las emociones y han reflexionado sobre la convivencia, la necesidad de abordar el pasado y el peligro que puede acarrear la no memoria" (Docente 8).

\section{Discusión de los resultados}

Partiendo de los resultados, y en relación al primer objetivo relacionado con el impacto ejercido por los testimonios, puede decirse que este ha sido grande y variado. Por un lado, han despertado un gran interés entre el alumnado, siendo motivadores y suscitando el interés de los/as estudiantes hacia el tema. Han permitido conocer una realidad desconocida para muchos/as, así como ampliar la mirada de aquellos que algo sabían, sobre todo en el caso de las personas que tenían sus ideas más asentadas. El impacto emocional ha sido enorme, además de suponer un aliciente importante para el desarrollo de la empatía. Y, por último, es destacable el poder del discurso de las víctimas, que ha tenido como consecuencia la asimilación de un discurso deslegitimizador de la violencia.

Entendemos que el interés despertado entre el alumnado se relaciona con el impacto emocional, el cual les ha permitido desarrollar una empatía hacia las víctimas y, como consecuencia, ampliar sus miradas en torno a un tema controvertido. Por lo tanto, reafirmamos la importancia que Arnoso et al. (2018) reconocen a la integración en el aula de testimonios silenciados tras un conflicto, a los cuales reconocen su función en la superación de visiones sesgadas de la realidad, en la que se presenta al "otro" como agresor y a uno mismo como víctima. En este caso, parte del alumnado ha escuchado de primera mano -algunos por primera vez-, además de los testimonios de las propias víctimas invitadas por el programa, las vivencias de sus familiares; y de segunda mano, las de los familiares de sus compañeros/as. Por lo tanto, la propuesta ha posibilitado 
contrastar diferentes puntos de vista, facilitando la construcción del discurso propio construido desde el espíritu crítico que reivindican Goldberg y Savenije (2018). En este caso, como señalan Bilbao y Etxeberria (2005), el punto de vista de las víctimas que participan en Adi-adian puede estimular procesos de reconciliación y desarrollar discursos no basados en la violencia, como se ha comprobado en este estudio, dando lugar a una transformación en la construcción de identidades (Marcos Macarro y Sánchez Medina, 2008).

En cuanto al segundo objetivo, que busca conocer si los testimonios son considerados patrimonio por el alumnado, no existen respuestas claras, pero sí algunos indicios. El alumnado ha mostrado un gran interés por conocer lo que ha pasado. Además, en los casos en los que no se le ha exigido, también ha hablado del tema con su entorno más cercano. Por lo tanto, parece que se ha desencadenado un proceso de patrimonialización (Fontal, 2003) hacia los testimonios. De esta manera, se ha generado un proceso de trasmisión entre generaciones, pero también entre iguales cuando han optado por hablar con amigos/as. Lucas y Estepa (2017) señalan que, cuanto más conocemos sobre un elemento patrimonial mayor implicación emocional se desencadena, pudiendo convertirlo en símbolo identitario digno de ser transmitido. Probablemente, en el caso del alumnado que ha contado lo escuchado en su entorno, el proceso de patrimonialización ha llegado a este grado. Igualmente, en tanto que hablamos de un tema controvertido, parece importante indicar que además de la trasmisión intergeneracional, también se intuye una patrimonialización del discurso del "otro", dando lugar a una memoria más amplia e inclusiva.

Desde el profesorado se identifica el tema como uno de los conflictos sociales candentes a tratar en el aula de ciencias sociales (López Facal y Santidrián, 2011), el cual ha sido muy bien recibido por parte del alumnado. Consideramos que el interés mostrado se debe a que es un tema presente en su día a día, pero sobre el que se les ha ocultado información. Siendo así, como señala Pineda-Alfonso (2015), creemos que utilizar temas controvertidos, supone una estrategia idónea para trabajar la ciudadanía y sus valores desde el respeto por los derechos humanos y el contraste de opiniones, permitiendo cuestionar la neutralidad de los individuos. Precisamente la complejidad de la neutralidad es una cuestión que preocupa al profesorado, especialmente en aquellos casos en los que existe una violación de los derechos fundamentales de las personas (Toledo Jofré et al., 2015). En este sentido, la experiencia desarrollada ha sido valorada positivamente por el profesorado en dos direcciones: una primera, de la que se destaca la posibilidad que ofrece para poner en marcha este tipo de proyectos en el aula; y una segunda, que otorga importancia a la introducción de estos temas en la formación inicial del profesorado, de cara a que cuenten con herramientas cuando sean docentes. Aunque de manera limitada, también se ha referenciado la posibilidad que el incorporar un tema/ conflicto al aula ofrece para abordar la gestión de conflictos, tan importante en la formación del profesorado.

Dicho esto, parecen claras las posibilidades señaladas en el tercer objetivo del estudio, por las que se reconoce la idoneidad del módulo educativo Adi-adian para trabajar las ciencias sociales a través de temas controvertidos y convertir la enseñanza de las ciencias sociales en una herramienta para el cambio social (Santisteban Fernández, 2019). Sin embargo, desde el profesorado se reconoce que se han aprovechado de manera parcial. Por un lado, porque se declara que el conocimiento sobre lo ocurrido no ha sido del todo el deseable, a falta de profundizar en el contexto histórico. Por otro, no se ha aprovechado para trabajar la introducción de temas controvertidos en el aula o la gestión de conflictos de manera teórica con los futuros docentes.

Para acabar, en relación al cuarto objetivo, se ha reconocido la implicación de las emociones en la propuesta, especialmente en relación a los testimonios, pero no así la posibilidad que ofrece para trabajar la educación emocional. Si bien se observa que, aun sin intencionalidad, se han dado procesos que podrían ser reconocidos como propuestas de educación emocional, son una excepción los/as docentes que los reconocen explícitamente. Interpretamos que esto puede deberse a la falta de conocimiento por parte del profesorado en torno a esta materia (Bidaurratzaga y Bustillo, 2020).

\section{Conclusiones}

Conscientes de la necesidad de profundizar en el estudio para llegar a conclusiones de mayor rotundidad, los primeros resultados señalan la buena dirección de la propuesta didáctica presentada.

El estudio que se ha llevado a cabo logra suplir la desinformación que el alumnado mostraba ante una etapa de su historia reciente y aumentar su motivación por conocer un conflicto social candente de su entorno: la vulneración de derechos humanos en el País Vasco durante las últimas décadas. El módulo educativo Adi-adian, y en especial los testimonios de las víctimas, han demostrado su eficacia para trabajar, a partir de un tema controvertido, cuestiones esenciales para el progreso de las sociedades democráticas, 
dando lugar a la formación de ciudadanos críticos y empáticos que abogan por la convivencia y deslegitiman la violencia.

El profesorado participante identifica la propuesta como una buena posibilidad para comenzar a introducir y reflexionar este tipo de temas en el aula. La enseñanza de las ciencias sociales debe promover el desarrollo de habilidades cívicas y de pensamiento crítico, además de erigirse en agente indispensable para el cambio social. En este sentido, destacamos el valor de los testimonios, en los cuales radica, en buena medida, el éxito de la implementación, ya que los discursos pronunciados a favor de la convivencia y la deslegitimación de la violencia a partir de realidades aparentemente contrapuestas han sido secundados por el alumnado. Este hecho parece haber favorecido un acercamiento al fenómeno histórico desde una mirada crítica, alejándose de posturas axiomáticas anteriores, producto de la experiencia personal. Además, otorgamos un valor patrimonial a los testimonios, que se han convertido en elementos de transmisión de la memoria colectiva.

Así, destacamos la importancia de incorporar el relato de las víctimas al aula, no solo por su valor informativo, sino por su impronta emocional. El impacto generado por los testimonios ha traído un creciente interés por conocer y comprender las circunstancias que se produjeron en el contexto narrado, llevando al alumnado hacia una identificación e implicación socio afectiva para con lo sucedido. En este sentido, reconocemos la importancia que las emociones desempeñan en la patrimonialización de los testimonios, los cuales son entendidos como vestigios de la memoria colectiva, transmisores del pasado y elementos simbólicos de la comunidad presente. Que el alumnado, tras haber escuchado a las víctimas, haya conversado sobre lo sucedido con sus allegados, pone de manifiesto el valor patrimonial de los testimonios, permitiendo al alumnado trascender lo aprendido en el aula a su vida diaria.

Como objeto de mejora, en tanto que la gestión de emociones y conflictos es parte del día a día del profesorado de las escuelas y, como tal, debiera formarse a nuestro alumnado en estas cuestiones, consideramos, por tanto, que en implementaciones futuras debería incluirse y profundizarse en la educación emocional y gestión de conflictos, cuyo potencial ha sido reconocido por parte del profesorado, pero no explotado. Por otro lado, el profesorado reconoce la importancia de abordar el contexto histórico de manera más profunda, posibilitando un mayor conocimiento de lo ocurrido que favorezca la interpretación de los hechos desde el pensamiento histórico.

Como conclusión final podemos decir que identificamos como patrimonio los testimonios de víctimas de violencia y destacamos su función como elemento patrimonializador de las memorias traumáticas. También identificamos su potencial emocional como elemento motivador y reconocemos su utilidad para introducir desde la empatía temas socialmente candentes en el aula, posibilitando el desarrollo de procesos de formación ciudadana.

\section{Apoyos}

Este proyecto ha contado con financiación del Vicerrectorado de Innovación, Compromiso Social y Acción Cultural de la Universidad del País Vasco (UPV-EHU), a través del Proyecto de Innovación Educativa Memoria y convivencia (HBP2019-20/102); y del Gobierno Vasco, a través del Grupo de Investigación GIPyPAC, UPV/EHU (IT1193-19).

\section{Referencias}

Arnoso, M., Páez, D., Bobowik, M. y Basabe, N. (2018). Implicaciones de los estudios sobre memoria colectiva para la enseñanza de la historia. Arbor, 194(788), a445. https://doi.org/10.3989/arbor.2018.788n2005

Bidaurratzaga, A. y Bustillo, J. (2020). Euskal Autonomi Erkidegoko Lehen Hezkuntzako irakasleen sinesmenak eta ezagutzak hezkuntza emozionalaren inguruan. IKASTORRATZA. e-Revista de Didáctica, 24, 1-15. DOI: $10.37261 / 24 \_a l e a / 1$

Bilbao, G. y Etxeberria, X. (2005). La presencia de las víctimas del terrorismo en la educación para la paz en el País Vasco. Bakeaz.

Carretero, M. (2007). Documentos de identidad. La construcción de la memoria histórica en un mundo global. Paidós.

Fontal Merillas, O. (2003). La educación patrimonial. Teoría y práctica en el aula, el museo e internet. Trea.

Fontal Merillas, O. y Martínez, M. (2017). Evaluación de programas educativos sobre Patrimonio Cultural Inmaterial. Estudios Pedagógicos, XLIII(4), 69-89.

Gobierno Vasco (2013). Módulo Educativo Adi-Adian. Aprendizajes de dignidad bumana, convivencia y empatía mediante una experiencia de escucha de testimonios de víctimas. Gobierno Vasco.

Goldberg, T. y Savenije, G.M. (2018). Teaching controversial historical issues. In S. A. Metzger y L. M. Harris (Eds.), The Wiley International Handbook of history teaching and learning (pp. 503-526). Wiley Blackwell. 
Gómez-Hurtado, I. y Cuenca López, J.M. (2017). Trabajar las emociones desde la educación patrimonial para atender a la diversidad del aula. En R. Martínez Media, R. García-Moris y C.R. García Ruiz (Eds.), Investigación en didáctica de las ciencias sociales. Reetos, preguntas y lineas de investigación (pp. 649-657). Universidad de Córdoba.

González, M. P. y Pagés, J. (2014). Historia, memoria y enseñanza de la historia: conceptos, debates y perspectivas europeas y latinoamericanas. Historia y Memoria, 9, 275-311.

López Facal, R. y Santidrián, V.M. (2011). Los «conflictos sociales candentes» en el aula. Iber: Didáctica de las ciencias sociales, geografía e historia, 69, 8-20.

López Noguero, F. (2002). El análisis de contenido como método de investigación. XXI Revista de Educación, 4, 167-179.

Lucas Palacios, L. y Estepa Giménez, J. (2017). Educación Patrimonial e Inteligencia Emocional. Hablan los alumnos. Clio: History and History Teacbing., 43. Recuperado de http://clio.rediris.es/n43/articulos/LucasEstepa2017.pdf

Marco Macarro, M.J. y Sánchez Medina, J.A. (2008). Memoria e identidad. Una aproximación desde la psicología actual La recuperación de la memoria histórica. Una perspectiva transversal desde las ciencais sociales (pp. 53-66). Fundación Centro de Estudios Andaluces.

Marcos Arévalo, J. (2010). El patrimonio como representación colectiva. La intangibilidad de los bienes culturales. Gazeta de Antropologia, 26(1).

Pagés, J., Martínez-Valcárcel, N. y Aldunate, M. C. (2014). El tiempo histórico: construcción y referentes en la memoria del alumnado. En J. Pagés, N. Martínez-Valcárcel y M. Cachari-Aldunate (Coords.), La historia de España en los recuerdos escolares: análisis e interpretación y poder de cambio de los testimonios de profesores $y$ alumnos (pp. 229-265). Nau Libres.

Pineda-Alfonso, J.A. (2015). Educar para la ciudadanía trabajando con temas controvertidos en Educación Secundaria Obligatoria. Revista de Investigación Educativa, 33(2), 353-367.

Rana, J., Willemsen, M. \& Dibbits, H.C. (2017). Moved by the tears of others: emotion networking in the heritage sphere. International Journal of Heritage Studies, 23(10), 977-988. doi:10.1080/13527258.2017.1362581

Santisteban Fernández, A. (2019). La enseñanza de las Ciencias Sociales a partir de problemas sociales o temas controvertidos: estado de la cuestión y resultados de una investigación. El Futuro del Pasado, 10, 57-79. doi:https://doi.org/10.14516/fdp.2019.010.001.002

Toledo Jofré, M.I., Magendzo, A., Gutiérrez Gianella, V. y Iglesias Segura, R. (2015). Enseñanza de 'temas controversiales' en la asignatura de historia y ciencias sociales desde la perspectiva de los profesores. Estudios Pedagógicos, 41(1), 275-292.

Usón González, I. (2017). Terrorismo y vulneraciones de Derechos Humanos de motivación política en el caso vasco: estudio exploratorio sobre los conocimientos y la valoración ética de la juventud universitaria vasca. Deusto Journal of Human Rights, 2, 121-148. http://dx.doi.org/10.18543/djhr-22017pp121-148

Wertsch, J., y Roediger, H. (2008). Collective memory: conceptual foundations and theoretical approaches. Memory, 16(3), 318-326. 